\title{
Genome-wide single-nucleotide polymorphism linkage analyses of quantitative rheumatoid arthritis phenotypes in Caucasian NARAC families
}

\author{
Kimberly E Taylor*1, Wei Chen², Christopher I Amos² and \\ Lindsey A Criswell ${ }^{1}$
}

Address: ${ }^{1}$ University of California, San Francisco, Rosalind Russell Medical Research Center for Arthritis, 374 Parnassus Avenue, Box 0500, San Francisco, California 94143, USA and 2Department of Epidemiology, University of Texas MD Anderson Cancer Center, 1155 Pressler Street, Unit 1340, Houston, Texas 77030, USA

Email: Kimberly E Taylor* - kim.taylor@ucsf.edu; Wei Chen - weichen@mdanderson.org; Christopher I Amos - camos@mdanderson.org; Lindsey A Criswell - lindsey.criswell@ucsf.edu

* Corresponding author

from Genetic Analysis Workshop 15

St. Pete Beach, Florida, USA. I I-I5 November 2006

Published: 18 December 2007

BMC Proceedings 2007, I (Suppl I):SI05

This article is available from: http://www.biomedcentral.com/I753-656I/I/SI/SI05

(C) 2007 Taylor et al; licensee BioMed Central Ltd.

This is an open access article distributed under the terms of the Creative Commons Attribution License (http://creativecommons.org/licenses/by/2.0),

which permits unrestricted use, distribution, and reproduction in any medium, provided the original work is properly cited.

\begin{abstract}
We applied nonparametric quantitative trait linkage analysis to two rheumatoid arthritis quantitative phenotypes, IgM rheumatoid factor (RF) and anti-cyclic citrullinated peptide autoantibody titer measurements, using 5700 genome-wide Illumina single-nucleotide polymorphism genotypes on 658 Caucasian North American Rheumatoid Arthritis Consortium families. Peak LOD scores for both quantitative traits were located in the human leukocyte antigen region 6p2I (I5.8 and I3.8 for RF and anti-cyclic citrullinated peptide, respectively) followed by I I I I (3.2 and 3.6). In addition, there were LOD scores of 3.2 on $2 q 32$ for RF and 3.6 on $4 q 24$ for anti-cyclic citrullinated peptide. The resulting linkage signals for both phenotypes are very similar to previous results for rheumatoid arthritis as a qualitative variable, with rheumatoid factor measurements being most closely aligned. Interestingly, anti-cyclic citrullinated peptide exhibits a stronger linkage peak on $2 \mathrm{pl} 4$ than rheumatoid factor and rheumatoid arthritis, and stronger linkage on $4 q 24$. Finally, we used ordered subset analyses to determine if sub-ranges of these two traits increased rheumatoid arthritis linkage signals; however, our analyses did not reveal significant effects of the quantitative traits on rheumatoid arthritis linkage signals in this population.
\end{abstract}




\section{Background}

Rheumatoid arthritis (RA) is a chronic autoimmune disease with heterogeneous phenotypes exhibited among affected individuals. While it is known to have a strong genetic component, studies attempting to identify chromosomal regions influencing RA have had mixed results, except for the human leukocyte antigen (HLA) region of chromosome 6 [1]. It is thought that the difficulty in identifying RA linkage regions may be due in part to its phenotypic heterogeneity, i.e., subtypes of this disease may have different genetic etiologies.

Recently, a genome-wide single-nucleotide polymorphism (SNP) analysis of North American Rheumatoid Arthritis Consortium (NARAC) families [2] implicated regions $2 \mathrm{q} 33$ and $11 \mathrm{p} 12$ in addition to the HLA region. Here we apply quantitative trait analysis to this same set of SNPs (provided as the Genetic Analysis Worskhop 15 (GAW15) Problem 2 data set), in order to explore genetic differences related to these phenotypes, and to take advantage of the potential increase in power of quantitative versus categorical analyses. In particular, we consider IgM rheumatoid factor (RF) and anti-cyclic citrullinated peptide (anti-CCP) autoantibody titer measurements, both of which are associated with RA but with incomplete and different specificities for the disease.

\section{Methods}

Families were selected for analysis that had at least two affected members with Illumina SNP genotyping and anti-CCP and/or RF titers. We also analyzed the Caucasian subgroup of families to limit genetic heterogeneity. PEDSTATS [3] was used to check for Mendelian inheritance errors, tabulate family structure, and evaluate HardyWeinberg equilibrium of the SNPs. MERLIN [4] was used for all linkage analyses (MINX, Merlin in X, for X chromosome analyses). Chromosomal positions were converted to centimorgans using the approximation of $1 \mathrm{Mb}=1 \mathrm{cM}$, which has been demonstrated to not significantly affect linkage signals [5]. Prior to analyses, unlikely genotypes were filtered by MERLIN, as described in online MERLIN documentation $[4,6]$.

Because linkage disequilibrium (LD) of tightly linked loci can lead to artificial inflation of linkage scores in the presence of some missing parental genotypes [7], regions of interest were analyzed using the MERLIN LD modelling feature. An $R^{2}$ value of 0.1 was used to define high-LD clusters.

Both quantitative traits were truncated - RF values below $11(16 \%)$ were set to 11 , and anti-CCP values above 210 (8\%) were set to 210 - because measurement of data in these ranges is not reliable (GAW15 Problem 2 notes). QT linkage analyses were performed using the MERLIN "-- deviates" option because both traits are not normally distributed and the NARAC sample is selected (in particular to contain multi-case RA families). This option necessitates specification of a population mean; in the absence of population data, we chose 11 as the mean for RF and 4.6 as the mean for anti-CCP, as done previously [8].

In order to directly compare the linkage results for RF and anti-CCP in regions of interest, we repeated the LD-modelled analyses using the subset of affected subjects having measurements for both quantitative traits.

Finally, anti-CCP and RF titers were evaluated for their influence on RA linkage on chromosomes 1 to 22 using the FLOSS (flexible ordered subset analysis) implementation [9] of ordered subset analysis [10]. The mean trait value of the family and the maximum difference among affected family members were both considered as ordering parameters to determine an optimal range of families for RA linkage evidence. FLOSS uses a Monte Carlo permutation test to compare the maximum ordered subset linkage score for this optimal set with the maximum obtained for random orderings of the families [9].

\section{Results}

A total of 1419 affected individuals from 658 families met our selection criteria, with 1415 having RF titers and 1341 having anti-CCP titers. Most families $(n=580)$ had a single sib pair meeting this criterion. In addition, there were 60 families with 3 affected members, 12 families with 4, 5 families with 5, and 1 family with 6 affected members. PEDSTATS reported a total of 814 sib pairs, 34 half-sib pairs, 11 cousins, 8 parent-child pairs, and 27 avuncular pairs. Of the affected individuals, $40 \%$ had one parent genotyped, and $10 \%$ had both parents genotyped.

PEDSTATS did not encounter any Mendelian errors in the available genotypes of these families. There were no HWE $p$-values below 0.0001, which we had chosen as the cutoff at which to remove SNPs; there was only one SNP (on chromosome 3 ) with $p<0.001$. Filtering of unlikely genotypes by MERLIN eliminated 5254 person-markers, $0.05 \%$ of the available genotypes.

With the resulting pedigrees, MERLIN (--deviates) was first run for all of chromosomes 1 through 22. MINX was used to analyze the $\mathrm{X}$ chromosomes. Then all chromosomes having peak LOD score $>3$ were rerun with MERLIN's LD cluster modeling $\left(R^{2}\right.$ value $\left.=0.1\right)$. The results of these peak locations with and without LD modeling are shown in Table 1. As in Amos et al. [2], the most substantial decrease when modeling LD was on chromosome 21. LOD scores over 3 with LD cluster modelling are in bold. 
Table I: Peak LOD scores and locations with/without LD clusters.

\begin{tabular}{|c|c|c|c|c|c|}
\hline \multirow[b]{2}{*}{ Trait } & \multirow[b]{2}{*}{ Chromosome } & \multicolumn{2}{|c|}{ Without LD clusters } & \multicolumn{2}{|c|}{ With LD clusters } \\
\hline & & Peak LOD & Locations (kb) & Peak LOD & Locations (kb) \\
\hline \multirow[t]{5}{*}{ Anti-CCP } & 2 & 3.1 & 70859 & 2.8 & 66769,67023 \\
\hline & 4 & 4.4 & 104210 & 3.6 & 104353 \\
\hline & 6 & 14.9 & 28965 & 13.8 & 32992 \\
\hline & 11 & 4.4 & 39183,39201 & 3.6 & 40486 \\
\hline & 21 & 8.3 & 37442,37455 & 2.4 & 40861 \\
\hline \multirow[t]{9}{*}{ RF } & 2 & 3.2 & 194143 & $3.1\left(2.3^{a}\right)$ & 194143 \\
\hline & 5 & 3.6 & 13709,13735 & 2.6 & 13722 \\
\hline & 6 & 18.4 & 28965 & I5.8 (14.4a) & 32992 \\
\hline & 10 & 3.4 & 60473,60475 & 2.6 & 75017 \\
\hline & 11 & 4 & 39201 & $3.2\left(4.2^{\mathrm{a}}\right)$ & 41024 \\
\hline & 12 & 3.7 & 131968,132017 & 1.6 & 22217 \\
\hline & 19 & 4.5 & 63636,63638 & 0.2 & 61193,61823 \\
\hline & 21 & 11.8 & $37433-37442$ & 1.5 & 40861 \\
\hline & $23-X$ & 3.2 & $56386-56493$ & 0.83 & 10450 \\
\hline
\end{tabular}

aUsing same set of subjects as anti-CCP analyses

In order to graphically compare the linkage curves of RF and anti-CCP titers on chromosomes 2, 4, 6, and 11, we repeated these analyses using only the affected subjects having both measurements. This kept the same families for anti-CCP, but reduced RF from 658 to 609 families (see footnoted items in Table 1). Figures 1, 2, 3, 4 show the resulting linkage curves.

Finally, we explored whether there were RF or anti-CCP trait-ordered subsets [10] that produced better evidence for RA linkage using FLOSS [9]. We used two per-family trait measures, the mean trait value and the maximum trait difference. Using this data set, we did not observe significant effects of the quantitative trait subsets on RA link-

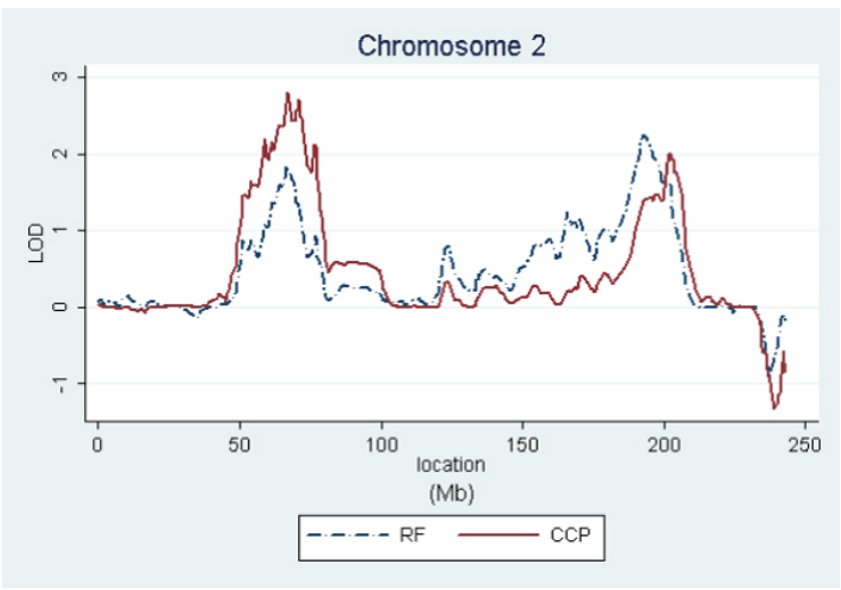

\section{Figure I}

RF and anti-CCP linkage on chromosome 2 for affected subjects having measurements for both traits. age signals. The most significant result $(p=0.04)$ was for the effect of RF maximum difference on linkage to chromosome 17, but with consideration for multiple testing (two traits for 22 chromosomes) this result does not provide convincing evidence for a non-random association with RA linkage.

\section{Discussion}

Our quantitative trait linkage results are similar to the RA linkage results using the same SNP set [2], with RF being most closely aligned to RA and anti-CCP exhibiting some interesting differences. For all three traits, the well established linkage peak in the HLA region on 6p21 extends far in the centromeric direction, extending into $6 \mathrm{q}$ and suggesting a possible secondary peak on 6q. A similar shape of lesser magnitude is exhibited by all three traits on chromosome 11p12. Linkage peak locations are also aligned on $4 \mathrm{q} 24$, but with the anti-CCP association being stronger (3.6 versus 2.3 using the same sample). On chromosome 2, RA and RF have the peak LOD score on 2q32-33, while the peak for anti-CCP is on $2 \mathrm{p} 14$. This difference in peaks is most pronounced in RA as described in Amos et al. [2]. Therefore, chromosome 2 may be interesting to explore further to understand the differences between RF and antiCCP and their relationships to RA etiologies.

Differences in linkage evidence compared with an earlier microsatellite quantitative trait locus (QTL) analysis of a subset of these families [8] - in particular on regions $2 \mathrm{p} 14,2 \mathrm{q} 32,4 \mathrm{q} 24$, and $11 \mathrm{p} 12$ - are very similar to differences in these two scans seen in RA linkage [2]. For RA, differences were attributed to the SNP scan having $44 \%$ higher information content, a larger set of families, and the possible heterogeneity of non-Caucasians included in 


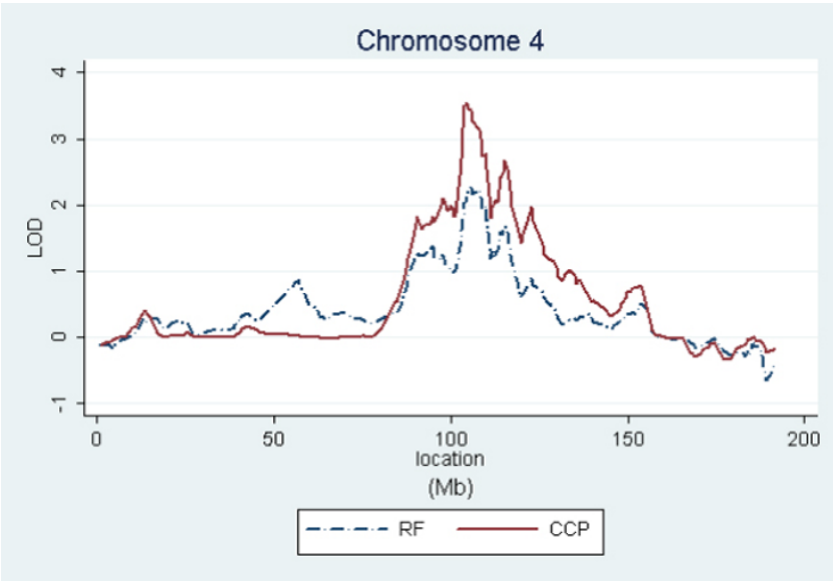

Figure 2

$\mathrm{RF}$ and anti-CCP linkage on chromosome 4 for affected subjects having measurements for both traits.

the microsatellite analyses [2]. Similarities between the RA and quantitative trait results are partially expected due to their correlation in the population, but are also addressed below.

The MERLIN deviates method was chosen for this analysis due to the non-normal trait distributions and because the trait sample was selected to only include RA subjects. We did not pursue variance-components [11] methods, including the Tobit VC method for censored data [12], because they assume normally distributed traits. However, when using the MERLIN deviates method, we have observed that single-point linkage scores for single sib pair families ( $88 \%$ of our selection) depend only on the direction of the quantitative trait from the mean. Because

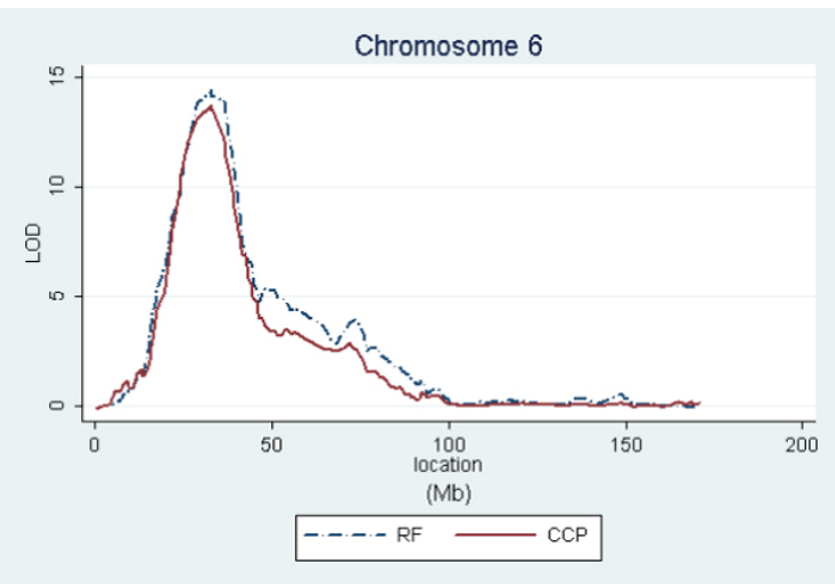

\section{Figure 3}

RF and anti-CCP linkage on chromosome 6 for affected subjects having measurements for both traits.

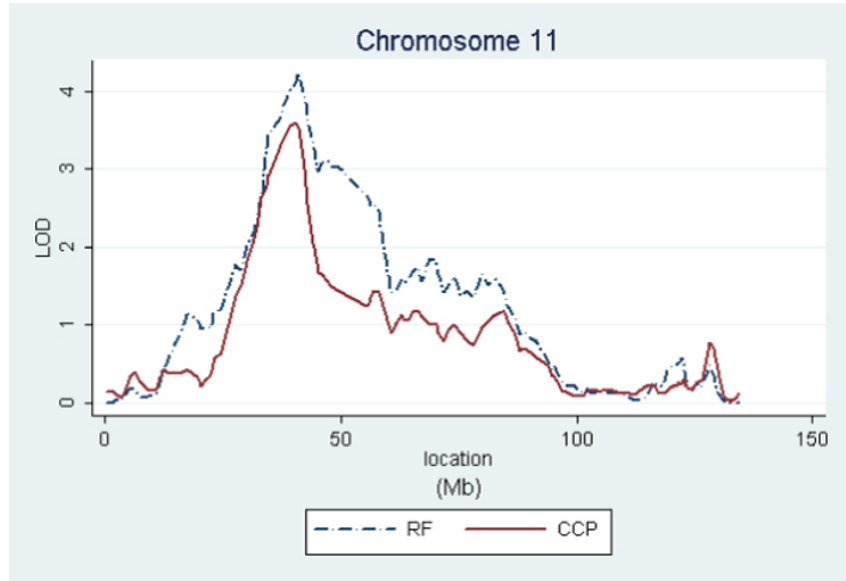

Figure 4

RF and anti-CCP linkage on chromosome II for affected subjects having measurements for both traits.

our selected RA sample is strictly above the RF population mean, and most are also above the CCP population mean, this reduces to RA linkage for a large portion of this data set. We believe that this partially explains the similarity in results between the RA and QTL linkage results for both our SNP analysis and the microsatellite analysis of [8]. We continue to investigate QTL methods that are appropriate for this selected non-normal data but can distinguish effects of the quantitative traits from RA.

Using ordered subset analyses, we did not identify any subset of families, based on quantitative trait ranges, which significantly increased the RA linkage signal. However, it is possible that this could be more successfully applied in a less selected population.

\section{Competing interests}

The author(s) declare that they have no competing interests.

\section{Acknowledgements}

We are grateful to the NARAC families for their participation and for the work of Dr. Peter Gregersen and the NARAC investigators. These studies were performed in part in the General Clinical Research Center, Moffitt Hospital, University of California, San Francisco, with funds provided by the National Center for Research Resources, 5 MOI RR00079, U.S. Public Health Service. This research is also supported in part by NIH grant ES09912.

This article has been published as part of BMC Proceedings Volume I Supplement I, 2007: Genetic Analysis Workshop 15: Gene Expression Analysis and Approaches to Detecting Multiple Functional Loci. The full contents of the supplement are available online at http://www.biomedcentral.com/ $|753-656| / \mid$ ? issue=SI. 


\section{References}

I. Fisher SA, Lanchbury JS, Lewis CM: Meta-analysis of four rheumatoid arthritis genome-wide linkage studies: confirmation of a susceptibility locus on chromosome I6. Arthritis Rheum 2003, 48: $1200-1206$.

2. Amos Cl, Chen WV, Lee A, Li W, Kern M, Lundsten R, Batliwalla F, Wener M, Remmers E, Kastner DA, Criswell LA, Seldin MF, Gregersen PK: High-density SNP analysis of 642 Caucasian families with rheumatoid arthritis identifies two new linkage regions on I I | I 2 and 2q33. Genes Immun 2006, 7:277-286.

3. Wigginton JE, Abecasis GR: PEDSTATS: descriptive statistics, graphics and quality assessment for gene mapping data. Bioinformatics 2005, 2 1:3445-3447.

4. Abecasis GR, Cherny SS, Cookson WO, Cardon LR: Merlin - rapid analysis of dense genetic maps using sparse gene flow trees. Nat Genet 2002, 30:97-101.

5. Ulgen A, Li W: Comparing single-nucleotide polymorphism marker-based and microsatellite marker-based linkage analyses. BMC Genet 2005, 6(SuppI I):SI3.

6. MERLIN online documentation [http://www.sph.umich.edu/csg/ abecasis/Merlin/]

7. Huang $\mathrm{Q}$, Shete $\mathrm{S}$, Amos $\mathrm{Cl}$ : Ignoring linkage disequilibrium among tightly linked markers induces false-positive evidence of linkage for affected sib pair analysis. Am J Hum Genet 2004, 75: I I06-III2.

8. Criswell LA, Chen WV, Jawaheer D, Lum RF, Wener MH, Gu X, Gregersen PK, Amos Cl: Dissecting the heterogeneity of rheumatoid arthritis through linkage analysis of quantitative traits. Arthritis Rheum 2007, 56:58-68.

9. Browning BL: FLOSS: flexible ordered subset analysis for linkage mapping of complex traits. Bioinformatics 2006, 22:5I2-5I3.

10. Hauser ER, Watanabe RM, Duren WL, Bass MP, Langefeld CD, Boehnke M: Ordered subset analysis in genetic linkage mapping of complex traits. Genet Epidemiol 2004, 27:53-63.

II. Amos C: Robust variance-components approach for assessing genetic linkage in pedigrees. Am J Hum Genet 1994, 54:535-543.

12. Epstein M, Lin X, Boehnke M: A Tobit variance-component method for linkage analysis of censored trait data. Am J Hum Genet 2003, 72:6I I-620.

Publish with Bio Med Central and every scientist can read your work free of charge

"BioMed Central will be the most significant development for disseminating the results of biomedical research in our lifetime. "

Sir Paul Nurse, Cancer Research UK

Your research papers will be:

- available free of charge to the entire biomedical community

- peer reviewed and published immediately upon acceptance

- cited in PubMed and archived on PubMed Central

- yours - you keep the copyright
BiolMedcentral 